\title{
Rank-into-rank hypotheses and the failure of $\mathrm{GCH}$
}

\author{
Vincenzo Dimonte, Sy-David Friedman*
}

December 11, 2013

\begin{abstract}
In this paper we are concerned about the ways $\mathrm{GCH}$ can fail in relation to rank-into-rank hypotheses, i.e., very large cardinals usually denoted by I3, I2, I1 and I0. The main results are a satisfactory analysis of the way the power function can vary on regular cardinals in the presence of rank-into-rank hypotheses and the consistency under I0 of the existence of $j: V_{\lambda+1} \prec V_{\lambda+1}$ with the failure of GCH at $\lambda$.

Keywords: Rank-into-rank, Embedding lifting, Easton forcing. 2010 Mathematics Subject Classifications: 03E55, 03E35 (03E45)
\end{abstract}

\section{Introduction}

The behaviour of the power function $\kappa \mapsto 2^{\kappa}$ has been under scrutiny since the birth of set theory. Already in 1878 Cantor proposed the Continuum Hypothesis and years later Hausdorff [9] extended it globally, stating for the first time the Generalized Continuum Hypothesis. Gödel's universe of constructible sets provided a model for the Generalized Continuum Hypothesis, while the consistency of its negation was first proved locally by Cohen and then globally by Easton [4], who used a class-product of Cohen forcings to prove the consistency of the failure of $\mathrm{GCH}$ at all regular cardinals. This left open the more difficult case of singular cardinals, known as the Singular

\footnotetext{
*E-mail addresses: vincenzo.dimonte@gmail.com (Vincenzo Dimonte), sdf@logic.univie.ac.at (Sy-David Friedman)

URLs: http://www.logic.univie.ac.at/ dimonte/ (Vincenzo Dimonte), http://www.logic.univie.ac.at/ sdf/ (Sy-David Friedman)

Kurt Gödel Research Center, University of Vienna, Währinger Straße 25, A-1090 Vienna, Austria
} 
Cardinal Problem. At the same time, another line of research was thriving. Just before the advent of forcing, Dana Scott [15] connected the power function with large cardinals, proving that if a measurable cardinal violates $\mathrm{GCH}$, then $\mathrm{GCH}$ is violated on a measure one set of cardinals below it. After that, Silver [16] proved that if the GCH holds below a singular cardinal of uncountable cofinality it must hold at that cardinal, and Solovay [17] proved that the GCH holds at all strong limit singular cardinals above a strongly compact cardinal. Therefore large cardinals have a big impact on the power function, and the investigation of the possible behaviours of the power function under large cardinal hypotheses is now a fundamental subject in the Singular Cardinal Problem analysis.

This paper is a contribution to the Singular Cardinal Problem in a novel case. After Kunen's proof that a Reinhardt cardinal is inconsistent [11], some very large cardinal hypotheses appeared, at the border of inconsistency, the rank-into-rank hypotheses I0-I3. These hypotheses naturally give rise to a strong limit cardinal of countable cofinality that is traditionally denoted as $\lambda$, and we study the failure of GCH at $\lambda$.

In Section 2 all the preliminary facts are collected. In Section 3 we present a proof of the consistency of I0 with the failure of GCH at regular cardinals. In Section 4 we use an absoluteness result of Woodin to prove that starting with a model of I0 there is a model of I1 in which the power function at $\lambda$ violates GCH in numerous ways. In Section 5 we extend the results of Section 4 to hypotheses stronger than I1.

The authors would like to thank the FWF (Austrian Science Fund) for its generous support through project P23316-N13 and the referee for her/his helpful suggestions.

\section{Preliminaries}

To avoid confusion or misunderstandings, all notation and standard basic results are collected here.

If $M$ and $N$ are sets or classes, $j: M \prec N$ denotes that $j$ is an elementary embedding from $M$ to $N$. We write the case in which the elementary embedding is the identity, i.e., $M$ is an elementary submodel of $N$, simply as $M \prec N$, while when $j$ is indicated we always suppose that it is not the identity.

If $j: M \prec N$ and either $M \vDash A C$ or $N \subseteq M$ then it moves at least one ordinal. The critical point, $\operatorname{crt}(j)$, is the least ordinal moved by $j$.

Let $j$ be an elementary embedding and $\kappa=\operatorname{crt}(j)$. Define $\kappa_{0}=\kappa$ and $\kappa_{n+1}=j\left(\kappa_{n}\right)$. Then $\left\langle\kappa_{n}: n \in \omega\right\rangle$ is the critical sequence of $j$. 
Kunen [11] proved under AC that if $M=N=V_{\eta}$ for some ordinal $\eta \leq$ Ord, and $\lambda$ is the supremum of the critical sequence, then $\eta$ cannot be bigger than $\lambda+1$ (and of course cannot be smaller than $\lambda$ ).

Kunen's result leaves room for a new breed of large cardinal hypotheses, sometimes referred to in the literature as rank-into-rank hypotheses:

I3 iff there exists $\lambda$ s.t. $\exists j: V_{\lambda} \prec V_{\lambda}$;

I2 iff there exists $\lambda$ s.t. $\exists j: V \prec M$, with $V_{\lambda} \subseteq M$ and $\lambda$ is the supremum of the critical sequence;

I1 iff there exists $\lambda$ s.t. $\exists j: V_{\lambda+1} \prec V_{\lambda+1}$.

The consistency order of the above hypotheses is reversed with respect to their numbering: I1 is strictly stronger than I2, which in turn is strictly stronger than I3 (see [12]). All of these hypotheses are strictly stronger than all of the large cardinal hypotheses outside the rank-into-rank umbrella (see [10], 24.9 for $n$-huge cardinals, or [1] for the Wholeness Axiom).

Note that if $j$ witnesses a rank-into-rank hypothesis, then $\lambda$ is uniquely determined by $j$, so in the following $\lambda$ always denotes the first nontrivial fixed point of the embedding $j$ under consideration.

An interesting point is that every elementary embedding $j: V_{\lambda} \prec V_{\lambda}$ has a unique extension to $V_{\lambda+1}$ : let $\left\langle\kappa_{n}: n \in \omega\right\rangle$ be its critical sequence; then for any $X \subseteq V_{\lambda}$ define $j^{+}(X)=\bigcup_{n \in \omega} j\left(X \cap V_{\kappa_{n}}\right)$. Then $j^{+}$is a $\Sigma_{0}$-elementary embedding from $V_{\lambda+1}$ to itself.

A consequence of this is the possibility of defining finite iterates of $j$, when $j: V_{\lambda} \prec V_{\lambda}$. Since $j \in V_{\lambda+1}$ and " $j: V_{\lambda} \prec V_{\lambda}$ is an elementary embedding" is a $\Sigma_{0}$ property of $V_{\lambda+1}$ with parameters $j, V_{\lambda}$, we can define $j_{2}=j^{+}(j)$, and consequently $j_{2}$ is an elementary embedding from $V_{\lambda}$ to itself. More generally, define $j_{n+1}=j^{+}\left(j_{n}\right)$. As for the iterates for I1-related elementary embeddings, Laver [12] proved that if $j: V_{\lambda+1} \prec V_{\lambda+1}$, then the extension of $j\left(j \uparrow V_{\lambda}\right)$ to $V_{\lambda+1}$ is in fact an elementary embedding, so we can define $j_{n+1}=\left(j\left(j_{n} \uparrow V_{\lambda}\right)\right)^{+}$. In the same paper Laver also proved a similar result for I2.

Suppose that $j$ witnesses I3 and let $\left\langle\kappa_{n}: n \in \omega\right\rangle$ be its critical sequence. Then it is immediate to see by elementarity that $\operatorname{crt}\left(j_{2}\right)=\operatorname{crt}\left(j^{+}(j)\right)=$ $j(\operatorname{crt}(j))=\kappa_{1}$, and more generally $\operatorname{crt}\left(j_{n+1}\right)=\kappa_{n}$. The same holds if $j$ witnesses I1: note that

$$
\left(j\left(j \uparrow V_{\lambda}\right)\right)^{+} \uparrow V_{\lambda}=\left(j \uparrow V_{\lambda}\right)^{+}\left(j \uparrow V_{\lambda}\right),
$$

so, as before, $\operatorname{crt}\left(j_{n+1}\right)=\operatorname{crt}\left(j_{n+1} \uparrow V_{\lambda}\right)=\kappa_{n}$. Therefore for any $\gamma<\lambda$ we can always assume $j$ to have a critical point between $\gamma$ and $\lambda$. 
We define also $j^{n}$ as the composition of $n$ copies of $j$, i.e., $j^{1}=j$ and $j^{n+1}=j \circ j^{n}$. Note that these are different from the iterates: for example, $j^{n}$ and $j$ have the same critical point, but $\operatorname{crt}\left(j_{2}\right)=j(\operatorname{crt}(j))$.

If $X$ is a set, then $L(X)$ denotes the smallest inner model that contains $X$; it is defined like $L$ but starting with the transitive closure of $\{X\}$ as $L_{0}(X)$.

In the early 1980's Woodin proposed an axiom even stronger than all the previous ones:

I0 For some $\lambda$ there exists a $j: L\left(V_{\lambda+1}\right) \prec L\left(V_{\lambda+1}\right)$, with $\operatorname{crt}(j)<\lambda$.

Note that if $\lambda$ witnesses I0, then $L\left(V_{\lambda+1}\right) \not \models$ AC, because otherwise $L\left(V_{\lambda+1}\right) \vDash$ ZFC, and we would contradict the proof of Kunen's Theorem [11], which shows that one cannot have $j: V \prec M$ with critical point less than $\lambda$ and a well-order of $V_{\lambda+1}$ in $V$. The fact that I0 is strictly stronger than I1 was proved by Laver [12].

Definition 2.1. We define $\Theta^{L\left(V_{\lambda+1}\right)}=\left\{\alpha: \exists \pi: V_{\lambda+1} \rightarrow \alpha, \pi \in L\left(V_{\lambda+1}\right)\right\}$, where $\rightarrow$ denotes a surjection.

The following is Lemma 5 in [18]:

Lemma 2.2. Let $j: L\left(V_{\lambda+1}\right) \prec L\left(V_{\lambda+1}\right)$ be such that $\operatorname{crt}(j)<\lambda$. Let

$$
U=U_{j}=\left\{X \in L\left(V_{\lambda+1}\right) \cap V_{\lambda+2}: j \uparrow V_{\lambda} \in j(X)\right\} .
$$

Then $U$ is an $L\left(V_{\lambda+1}\right)$-ultrafilter such that $\operatorname{Ult}\left(L\left(V_{\lambda+1}\right), U\right)$ is well-founded. By condensation the collapse of $\operatorname{Ult}\left(L\left(V_{\lambda+1}\right), U\right)$ is $L\left(V_{\lambda+1}\right)$, and $j_{U}: L\left(V_{\lambda+1}\right) \prec$ $L\left(V_{\lambda+1}\right)$, the inverse of the collapse, is an elementary embedding. Moreover, there is an elementary embedding $k_{U}: L\left(V_{\lambda+1}\right) \prec L\left(V_{\lambda+1}\right)$ with $\operatorname{crt}\left(k_{U}\right)>$ $\Theta^{L\left(V_{\lambda+1}\right)}$ such that $j=k_{U} \circ j_{U}$.

Definition 2.3. Let $j: L\left(V_{\lambda+1}\right) \prec L\left(V_{\lambda+1}\right)$ be such that $\operatorname{crt}(j)<\lambda$. Then $j$ is proper iff $j=j_{U_{j}}$.

By Lemma 2.2 any elementary embedding $j: L\left(V_{\lambda+1}\right) \prec L\left(V_{\lambda+1}\right)$ can be substituted with a proper one, that coincides on $L_{\Theta^{L\left(V_{\lambda+1}\right)}}\left(V_{\lambda+1}\right)$.

Properness is used to define finite iterates for the I0 case: let $j: L\left(V_{\lambda+1}\right) \prec$ $L\left(V_{\lambda+1}\right)$ with $\operatorname{crt}(j)<\lambda$ be a proper elementary embedding, and let $U=U_{j}$ be the relevant ultrafilter. Define

$$
j(U)=\bigcup\left\{j(\operatorname{ran}(\pi)): \pi \in L\left(V_{\lambda+1}\right), \pi: V_{\lambda+1} \rightarrow U\right\}
$$

and then define $j_{2}$ as the map associated to $j(U)$. All the $j_{n}$ are defined accordingly by induction. Woodin [18] proved that the $j_{n}$ are elementary 
embeddings, so also in this case for any $\gamma<\lambda$ we can always assume $j$ to have a critical point between $\gamma$ and $\lambda$.

The elements of the critical sequence of elementary embeddings that witness rank-into-rank hypotheses are really large cardinals.

Definition 2.4. Let $\kappa$ be an uncountable cardinal. We say that $\kappa$ is:

- strong limit if for any $\eta<\kappa, 2^{\eta}<\kappa$;

- measurable if there is a nontrivial $\kappa$-complete ultrafilter over $\kappa$;

- strongly compact if for any $\eta \geq \kappa$ there is a fine $\kappa$-additive measure on $\mathcal{P}_{\kappa}(\eta)$, where $U$ is fine means that for any $\xi<\eta,\{A: \xi \in A\} \in U$;

- supercompact if for any $\eta \geq \kappa$ there is a fine normal measure on $\mathcal{P}_{\kappa}(\eta)$, i.e., a measure $U$ such that for any $f: \mathcal{P}_{\kappa}(\eta) \rightarrow \eta$ such that $f(x) \in x$ for almost every $x, f$ is constant on a set in $U$;

- $n$-huge if there is a sequence $\kappa=\lambda_{0}<\lambda_{1}<\cdots<\lambda_{n}=\lambda$ and a $\kappa$-complete normal ultrafilter $U$ over $\mathcal{P}(\lambda)$ such that for any $i<n$,

$$
\left\{x \in \mathcal{P}(\lambda): \operatorname{ot}\left(x \cap \lambda_{i+1}\right)=\lambda_{i}\right\} \in U .
$$

Let $j: V_{\lambda} \prec V_{\lambda}$ and let $\left\langle\kappa_{n}: n \in \omega\right\rangle$ be its critical sequence. Note that for any $n, \kappa_{n}=\operatorname{crt}\left(j_{n+1}\right)$, so any property of $\kappa_{0}$ expressible in $V_{\lambda}$ is shared by all of the $\kappa_{n}$ 's. We have

- " $\kappa_{0}$ is measurable" is witnessed by $U=\left\{X \subseteq \kappa_{0}: \kappa_{0} \in j(X)\right\}$;

- " $\kappa_{0}$ is $n$-huge" is witnessed by $\kappa_{0}<\kappa_{1}<\cdots<\kappa_{n}$ and

$$
U=\left\{X \subseteq \mathcal{P}\left(\kappa_{n}\right): j^{\prime \prime} \kappa_{n} \in j(X)\right\} ;
$$

- " $V_{\lambda} \vDash \kappa_{0}$ is strongly compact and supercompact": let $\kappa_{0} \leq \eta<\lambda$, and suppose $\eta<\kappa_{n}$; then this is witnessed by

$$
U=\left\{X \subseteq \mathcal{P}_{\kappa_{0}}(\eta):\left(j^{n}\right)^{\prime \prime} \eta \in j^{n}(X)\right\}
$$

The situation is radically different for $\lambda$, since it is a singular cardinal, and the large cardinals described in Definition 2.4 (strong limit excluded) must be regular. However, since it is a limit of strong limit cardinals, $\lambda$ is a strong limit cardinal. Moreover, we trivially have that $V_{\kappa_{0}} \prec V_{\kappa_{1}}$ and for any $n \in \omega, V_{\kappa_{n}} \prec V_{\kappa_{n+1}}$. But then the $V_{\kappa_{n}}$ 's form a direct system with limit $V_{\lambda}$ and $V_{\kappa_{n}} \prec V_{\lambda}$ for any $i \in \omega$. In particular, $V_{\lambda}$ is a model of ZFC. 
For proving results about rank-into-rank elementary embeddings and the continuum function two forcings will be used: Easton forcing, that will be explained in the following section, and Prikry forcing. For both forcings we use the notation $\check{a}$ to indicate the canonical name of an element in the ground model, and $\dot{G}$ to indicate the canonical name for the generic.

Prikry forcing (a detailed discussion about it can be found in [7]) is defined as follows: fix an ultrafilter $U$ over $\kappa$ measurable; $p \in \mathbb{P}$ iff $p=(s, A)$, where $s \in[\kappa]^{<\omega}, A \in U$ and $\bigcup s<\bigcap A$. For $p=(s, A), q=(t, B) \in \mathbb{P}$, we say $q \leq p$ iff $s \subseteq t, B \subseteq A$ and $t \backslash s \subseteq A$.

Theorem 2.5. Prikry forcing on $\kappa$ is $\kappa^{+}$-c.c. and doesn't add bounded subsets of $\kappa$.

\section{$3 \quad \mathrm{GCH}$ and its negation at regular cardinals}

There are various results already published about the interaction of rankinto-rank hypotheses with different behaviours of the continuum function. The following definition captures the concept of "right behaviour" of the continuum function at regular cardinals:

Definition 3.1. Let $E:$ Reg $\rightarrow$ Card be a class function. Then $E$ is an Easton function iff

- $\alpha<\beta \rightarrow E(\alpha) \leq E(\beta)$;

- $\operatorname{cof}(E(\alpha))>\alpha$ for all $\alpha \in$ Reg.

Theorem 3.2. Let $I^{*}$ be I3, I2, I1 or I0, and suppose that $I^{*}$ holds with associated cardinal $\lambda$. Let $E$ be an Easton function such that $E \uparrow \lambda$ is definable over $V_{\lambda}$. Then in a generic extension, $I^{*}$ still holds for $\lambda$ and $2^{\kappa}=E(\kappa)$ for regular $\kappa$. Moreover, if $E(\kappa)=\kappa^{+}$for regular $\kappa$ we have

$$
\mathrm{Con}\left(\mathrm{ZFC}+I^{*}\right) \rightarrow \operatorname{Con}\left(\mathrm{ZFC}+I^{*}+\mathrm{GCH}\right) .
$$

Note that in the I0 case the Easton function will be realized in $V[G]$, not in $L\left(V_{\lambda+1}\right)[G]$ or $L\left(V[G]_{\lambda+1}\right)$.

Specific cases of Theorem 3.2 have already appeared in the literature: Hamkins [8] proved it for I1 and $E(\kappa)<2^{\kappa}$, Corazza [2] proved it for I3 and $E(\kappa)$ less than the first inaccessible cardinal larger than $\kappa$, and Friedman [5] proved it for I2 and GCH. Although the framework of the proof is the same as in the cited papers, Theorem 3.2 weakens the hypotheses on $E$ and also considers I0. For this reason, we give the proof just for the I0 case. 
The main tool for the proof is forcing iteration. If $\mathbb{P}_{\lambda}$ is a forcing iteration of length $\lambda$, for any $\delta<\lambda$ we denote $\mathbb{P}_{\delta}$ as the $\delta$-th stage of the iteration, and $\mathbb{Q}_{\delta}$ as the $\delta$-th forcing of the iteration.

Definition 3.3. Let $\mathbb{P}_{\lambda}$ be a forcing iteration of length $\lambda$, where $\lambda$ is either a strong limit cardinal or is equal to $\infty$, the class of all ordinals. We say that $\mathbb{P}_{\lambda}$ is

- reverse Easton if nontrivial forcing is done only at infinite cardinal stages, direct limits are taken at all inaccessible cardinal limit stages, and inverse limits are taken at all other limit stages; moreover, $\mathbb{P}_{\lambda}$ is the direct limit of the $\left\langle\mathbb{P}_{\delta}, \delta<\lambda\right\rangle$ if $\lambda$ is regular or $\infty$, the inverse limit of the $\left\langle\mathbb{P}_{\delta}, \delta<\lambda\right\rangle$, otherwise;

- directed closed if for all $\delta<\lambda, \mathbb{Q}_{\delta}$ is $<\delta$-directed closed, i.e., for any $D \subseteq \mathbb{Q}_{\delta},|D|<\delta$ such that for any $d_{1}, d_{2} \in D$ there is an $e \in D$ with $e \leq d_{1}, e \leq d_{2}$, there exists $p \in \mathbb{Q}_{\delta}$ such that $p \leq d$ for any $d \in D$;

- $\lambda$-bounded if for all $\delta<\lambda, \mathbb{Q}_{\delta}$ has size $<\lambda$. Note that in the case $\lambda=\infty$, this just means that each $\mathbb{Q}_{\delta}$ is a set-forcing;

- above $\omega$ if $\mathbb{Q}_{\omega}$ is trivial forcing.

Moreover, if $j$ is any elementary embedding such that $j^{\prime \prime} \lambda \subset \lambda$ and $\mathbb{P}_{\lambda} \subset$ $\operatorname{dom}(j)$, we say that $\mathbb{P}_{\lambda}$ is $j$-coherent if for any $\delta<\lambda, j\left(\mathbb{P}_{\delta}\right)=\mathbb{P}_{j(\delta)}$.

Note that these definitions are local, i.e., if $\mathbb{P}_{\lambda}$ satisfies one of the above, then for any $\delta<\lambda, \mathbb{P}_{\delta}$ satisfies the same.

The following theorem was proved by Easton in 1970:

Theorem 3.4 (Easton, [4]). Let $E$ be an Easton function. Then there exists a generic extension $V[G]$ of $V$ such that $V[G] \vDash \forall \kappa\left(\kappa\right.$ regular $\left.\rightarrow 2^{\kappa}=E(\kappa)\right)$.

Theorem 3.4 can be proved using iterated forcing. Define $\mathbb{Q}_{\delta}$, for $\delta$ closed under $E$, as the Easton product of forcings to make $2^{\gamma}=E(\gamma)$ for regular $\gamma$ in $\left[\delta, \delta^{*}\right)$, where $\delta^{*}$ is the least cardinal greater than $\delta$ closed under $E$. Limits are taken so that the iteration is reverse Easton. It is easy to prove that this forcing is directed closed. If $\lambda$ is closed under $E$ then the forcing is $\lambda$-bounded, and $\left\langle\mathbb{P}_{\alpha}: \alpha<\lambda\right\rangle \subseteq V_{\lambda}$. Finally, if $E \uparrow \lambda$ is definable over $V_{\lambda}$, then $\left\langle\mathbb{P}_{\alpha}: \alpha<\lambda\right\rangle$ is definable over $V_{\lambda}$ and if the parameters used in the definition of $E \uparrow \lambda$ are in $V_{\operatorname{crt}(j)}$ then $\mathbb{P}_{\lambda}$ is $j$-coherent.

We will prove the following: 
Theorem 3.5. Let $j: L\left(V_{\lambda+1}\right) \prec L\left(V_{\lambda+1}\right)$ with $\operatorname{crt}(j)<\lambda$ and $\mathbb{P}_{\lambda} a \lambda$ bounded, $j$-coherent, directed closed, reverse Easton iteration. Then for $\mathbb{P}_{\lambda^{-}}$ generic $G, j$ lifts to $j^{*}: L\left(V_{\lambda+1}\right)[G] \prec L\left(V_{\lambda+1}\right)[G]$ and the restriction of such a lifting to $L\left(V[G]_{\lambda+1}\right)$ witnesses $I 0$ in $V[G]$.

Lemma 3.6. Let $j: L\left(V_{\lambda+1}\right) \prec L\left(V_{\lambda+1}\right)$ with $\operatorname{crt}(j)<\lambda$ and $\mathbb{P}_{\lambda}$ a $\lambda$-bounded, $j$-coherent, directed closed, reverse Easton iteration. Then there exists $q \in \mathbb{P}_{\lambda}$ such that $q \Vdash p \in \dot{G} \rightarrow j(p) \in \dot{G}$.

Proof. Let $\left\langle\kappa_{n}: n \in \omega\right\rangle$ be the critical sequence of $j$. The key point is that $j$-coherence limits the largeness of forcing stages. Let $\gamma<\kappa_{0}$. Then if $\left|\mathbb{P}_{\gamma}\right| \geq \kappa_{0}$, by elementarity we would have $\left|j\left(\mathbb{P}_{\gamma}\right)\right|=\left|\mathbb{P}_{j(\gamma)}\right|=\left|\mathbb{P}_{\gamma}\right| \geq \kappa_{1}$, and by $\omega$ applications of $j$ it follows $\left|\mathbb{P}_{\gamma}\right| \geq \lambda$, a contradiction by $\lambda$-boundedness. By elementarity, it follows that for any $\gamma<\kappa_{n},\left|\mathbb{P}_{\gamma}\right|<\kappa_{n}$.

We define $q$ piece by piece. Let $q \uparrow \kappa_{1}$ be the trivial condition. Now, fix $n \geq 1$. Then

$$
\Vdash\left|\left\{j(p)\left(\kappa_{n}\right): p \in \dot{G}\right\}\right|<\kappa_{n},
$$

because

$$
\begin{aligned}
& \left\{j(p)\left(\kappa_{n}\right): p \in G\right\}=\left\{j\left(p\left(\kappa_{n-1}\right)\right): p \in G\right\}= \\
& \quad=\left\{j\left(p\left(\kappa_{n-1}\right)\right): p\left\lceil\left(\kappa_{n-1}\right)^{+} \in G \cap \mathbb{P}_{\left(\kappa_{n-1}\right)^{+}}\right\}\right.
\end{aligned}
$$

and $\left|\mathbb{P}_{\left(\kappa_{n-1}\right)^{+}}\right|<\kappa_{n}$. So, since $\mathbb{Q}_{\kappa_{n}}$ is $<\kappa_{n}$-directed closed in $V^{\mathbb{P}_{\kappa_{n}}}$, there exists a name $\tau$ such that $\Vdash \forall p \in \dot{G} \tau \leq j(p)\left(\kappa_{n}\right)$, and define $q\left(\kappa_{n}\right)=\tau$. As for the definition of $q$ between elements of the critical sequence, we have that $\Vdash \mid j^{\prime \prime} \dot{G}\left\lceil\left(\kappa_{n}, \kappa_{n+1}\right) \mid \leq \kappa_{n}\right.$, and as $\mathbb{P}_{\kappa_{n}, \kappa_{n+1}}$ (the forcing strictly between $\kappa_{n}$ and $\left.\kappa_{n+1}\right)$ is $\leq \kappa_{n}$-directed closed there exists a name $\tau$ such that

$$
\Vdash \forall p \in \dot{G} \forall \beta \in\left(\kappa_{n}, \kappa_{n+1}\right) \tau(\beta) \leq j(p)(\beta)
$$

and define $q \uparrow\left(\kappa_{n}, \kappa_{n+1}\right)=\tau$. Now, suppose that $p, q \in G$. Clearly $q \uparrow$ $\left[\kappa_{1}, \lambda\right) \leq j(p) \uparrow\left[\kappa_{1}, \lambda\right)$, so $j(p) \uparrow\left[\kappa_{1}, \lambda\right) \in G \uparrow\left[\kappa_{1}, \lambda\right)$. But $j(p) \uparrow \kappa_{0}=p$, and $j(p) \uparrow\left[\kappa_{0}, \kappa_{1}\right)$ is trivial, so $j(p) \in G$.

To use the typical lifting lemma, we have to prove that in fact the model $L\left(V_{\lambda+1}\right)$ as constructed in the generic extension is in the domain of the lifting.

Lemma $3.7([8])$. If $\mathbb{P}_{\lambda}$ is a $\lambda$-bounded, directed closed forcing iteration, then $V[G]_{\lambda+1}=V_{\lambda+1}[G]$. 
Proof. Let $p$ be a condition forcing that $\sigma$ is a name for a subset of $\lambda$. Then there is an extension $q$ of $p$ and a sequence $\left\langle\sigma_{n}: n<\omega\right\rangle$ in $V$ such that for each $n, q$ forces $\sigma \cap \kappa_{n}=\sigma_{n}$ and $\sigma_{n}$ is a canonical $\mathbb{P}_{\kappa_{n}}$-name for a subset of $\kappa_{n}$. Then each $\sigma_{n}$ belongs to $V_{\lambda}$ and therefore $q$ forces $\sigma=\sigma^{*}$ for some name $\sigma^{*}$ in $V_{\lambda+1}$. This proves that $V[G]_{\lambda+1}$ is contained in $V_{\lambda+1}\left[G \cap V_{\lambda}\right]=V_{\lambda+1}[G]$. The converse is clear, as any element of $V_{\lambda+1}[G]$ belongs to $L\left(X, G \cap V_{\lambda}\right)$ for some $X$ in $V_{\lambda+1}$ and therefore belongs to $V[G]_{\lambda+1}$.

Although it will not be used in this paper, the following Corollary of Lemma 3.7 is of independent interest.

Corollary 3.8. If $\mathbb{P}_{\lambda}$ is a $\lambda$-bounded, directed closed, reverse Easton iteration, and $G$ is $\mathbb{P}_{\lambda}$-generic then $L\left(V[G]_{\lambda+1}\right)$ is contained in $L\left(V_{\lambda+1}\right)[G]$. If $\mathbb{P}_{\lambda}$ is above $\omega$ then we have equality.

Proof. The first conclusion follows immediately from Lemma 3.7 by induction, it is possible to prove that a set constructible in $V[G]$ from some elements has a name that is constructible in $V$ from the names of such elements, therefore by Lemma 3.7 any element of $L\left(V[G]_{\lambda+1}\right)$ has a name in $L\left(V_{\lambda+1}\right)$.

For the second conclusion, note that if $\mathbb{P}_{\lambda}$ is above $\omega$ and $X$ is a subset of $\lambda$ in $V[G]$ then $X$ belongs to $V$ iff $X \cap \kappa_{n}$ belongs to $V$ for each $n$, and therefore as elements of $V_{\lambda+1}$ are coded by subsets of $\lambda, V_{\lambda+1}$ belongs to $L\left(V_{\lambda}, V[G]_{\lambda+1}\right)=L\left(V[G]_{\lambda+1}\right)$. From this it follows that $G$ also belongs to $L\left(V[G]_{\lambda+1}\right)$ as $p \in G$ iff $p \in V_{\lambda+1}$ and $p \mid V_{\alpha} \in G \cap V_{\lambda}$ for all $\alpha<\lambda$, and so $G$ belongs to $L\left(V_{\lambda+1}, G \cap V_{\lambda}\right)$. As both $V_{\lambda+1}$ and $G$ belong to $L\left(V[G]_{\lambda+1}\right)$ we can then conclude that $L\left(V_{\lambda+1}\right)[G]$ is contained in $L\left(V[G]_{\lambda+1}\right)$.

Now we have enough tools to prove Theorem 3.5 .

Proof of Theorem 3.5. Let $j: L\left(V_{\lambda+1}\right) \prec L\left(V_{\lambda+1}\right)$ with $\operatorname{crt}(j)<\lambda$. We can suppose that the condition $q$ in Lemma 3.6 is in the generic $G$. By the usual argument, we can extend $j$ to $j^{\prime}: L\left(V_{\lambda+1}\right)[G] \prec L\left(V_{\lambda+1}\right)[G]$, letting $j\left(\tau_{G}\right)=j(\tau)_{G}$ for any $\tau \in L\left(V_{\lambda+1}\right)$, and $j^{\prime}$ is an elementary embedding. By Corollary 3.8 that $L\left(V[G]_{\lambda+1}\right) \subseteq L\left(V_{\lambda+1}\right)[G]$, and as $j^{\prime}\left(V[G]_{\lambda+1}\right)=V[G]_{\lambda+1}$, the restriction of $j^{\prime}$ to $L\left(V[G]_{\lambda+1}\right)$ witnesses I0 in $V[G]$.

Corollary 3.9. Suppose $I 0$ and let $E$ be an Easton function such that $E \uparrow \lambda$ is definable over $V_{\lambda}$. Then $\mathrm{Con}(\mathrm{ZFC}+I 0+\mathrm{GCH})$ and there is a generic extension that satisfies $I 0+2^{\kappa}=E(\kappa)$ holds for all regular $\kappa$.

Proof. Fix a $j: L\left(V_{\lambda+1}\right) \prec L\left(V_{\lambda+1}\right)$ with $\operatorname{crt}(j)<\lambda$ and such that all the parameters that define $E \uparrow \lambda$ are in $V_{\operatorname{crt}(j)}$. Let $\mathbb{P}$ be the Easton iteration on all ordinals from Theorem 3.4. First we consider the first $\lambda$ steps of the 
iteration, $\mathbb{P}_{\lambda}$. Then $\left\langle\mathbb{P}_{\alpha}: \alpha<\lambda\right\rangle \subseteq V_{\lambda}$ is definable in $V_{\lambda}$ with parameters that are fixed points of $j$, therefore $\mathbb{P}_{\lambda}$ is $\lambda$-bounded and $j$-coherent, so by Theorem $3.5 j$ lifts to

$$
j^{\prime}: L\left(V\left[G \cap V_{\lambda}\right]_{\lambda+1}\right) \prec L\left(V\left[G \cap V_{\lambda}\right]_{\lambda+1}\right) .
$$

Now, the rest of the iteration is $<\lambda^{+}$-directed closed, in particular is $\lambda$ closed (every descending sequence of length $\lambda$ has a lower bound), therefore it doesn't change $L\left(V[G]_{\lambda+1}\right)$, and $j^{\prime}$ witnesses I0 in $V[G]$.

After having proved the consistency of rank-into-rank hypotheses with the failure of GCH at regular cardinals, the next step is to prove it at some singular cardinal. However, there are some well-known limitations:

Theorem 3.10 (Solovay [17]). Let $\kappa$ be a strongly compact cardinal. Let $\lambda$ be a singular strong limit cardinal greater than $\kappa$. Then $2^{\lambda}=\lambda^{+}$.

Suppose that $j: V_{\lambda} \prec V_{\lambda}$. The critical point of $j$ is strongly compact in $V_{\lambda}$, so for any $\operatorname{crt}(j)<\eta<\lambda$ singular strong limit cardinal we have $2^{\eta}=\eta^{+}$, and this is impossible to kill while preserving the embedding. For the rest of this article we will focus on the failure of $\mathrm{GCH}$ at $\lambda$, the first point not covered by Solovay's result.

Theorem 3.11. Let $j: V_{\lambda} \prec V_{\lambda}$. Then for any $\delta<\aleph_{1}$ there exists a generic extension $V[G]$ such that $j$ lifts to $V[G]_{\lambda}$ and $2^{\lambda}=\lambda^{+\delta+1}$.

This is a simple corollary of Gitik's construction using short extenders for blowing up the size of the power set of a singular cardinal [6]:

Theorem 3.12. Assume GCH. Suppose $\lambda$ is a cardinal of cofinality $\omega$ such that $\left\{\kappa<\lambda: o(\kappa) \geq \kappa^{+n}\right\}$ is cofinal in $\lambda$ for every $n \in \omega$. Then for any $\delta<\aleph_{1}$ there exist a cofinality preserving generic extension having the same bounded subsets of $\lambda$ and satisfying $2^{\lambda}=\lambda^{+\delta+1}$.

Proof of Theorem 3.11. Let $j: V_{\lambda} \prec V_{\lambda}$ and $\left(\kappa_{n}\right)_{n \in \omega}$ the critical sequence. Then for any $n, m \in \omega, o\left(\kappa_{n}\right) \geq\left(\kappa_{n}\right)^{+m}$, therefore $\lambda$ satisfies the hypotheses of Theorem 3.12. Moreover, by Theorem 3.2 we can suppose GCH, thereby allowing the proof of Theorem 3.12 to go through. Since Gitik's forcing doesn't add bounded subsets of $\lambda$, we have trivially $V_{\lambda}=V[G]_{\lambda}$, so there is no need to lift $j$, and Theorem 3.11 is proved.

The case I2 has been treated by Cummings and Foreman: in [3] they prove, using a hypothesis slightly stronger than $\mathrm{I} 2$, that $\mathrm{I} 2(\lambda)$ is consistent with $2^{\lambda}=\lambda^{++}$. 
Unfortunately, the same cannot be said for I1. There are many obstacles for lifting an I1 elementary embedding to a forcing that kills GCH at $\lambda$, like the fact that the names for elements of $V[G]_{\lambda+1}$ live outside $V_{\lambda+1}$, or the difficulties of finding a master condition for such a forcing. This is why we change strategy, and we reflect the embedding instead of lifting it. For this we need more information about elementary embeddings that witness I0.

\section{Consistency of I1 and the negation of GCH}

In the Preliminaries we defined the finite iterates for an I0 elementary embedding, but in this section we need more.

Definition 4.1. Let $j: L\left(V_{\lambda+1}\right) \prec L\left(V_{\lambda+1}\right)$ with $\operatorname{crt}(j)<\lambda$ be a proper elementary embedding, and let $U=U_{j}$ be the relevant ultrafilter. Remember that the second iterate of $j$ was defined as the map associated to $j(U)$.

Define the successive iterates in the usual way: let $\alpha$ be an ordinal. Then

- if $\alpha=\beta+1, M_{\beta}$ is well-founded and $j_{\beta}: M_{\beta} \prec M_{\beta}$ is the ultrapower via $W$, then $M_{\alpha}=\operatorname{Ult}\left(M_{\beta}, j_{\beta}(W)\right)$ and $j_{\alpha}=j_{\beta}\left(j_{\beta}\right)$.

- if $\alpha$ is a limit, let $\left(M_{\alpha}, j_{\alpha}\right)$ be the direct limit of $\left(M_{\beta}, j_{\beta}\right)$ with $\beta<\alpha$.

We say that $j$ is iterable, if for every $\alpha \in \mathrm{Ord}, M_{\alpha}$ is well-founded and $j_{\alpha}: M_{\alpha} \prec M_{\alpha}$. In this case, we call $j_{\alpha, \beta}$ the natural embeddings between $M_{\alpha}$ and $M_{\beta}$.

The following is a conjunction of Lemma 16 and Lemma 21 in [18]:

Theorem 4.2. Let $j: L\left(V_{\lambda+1}\right) \prec L\left(V_{\lambda+1}\right)$ with $\operatorname{crt}(j)<\lambda$ be a proper elementary embedding. Then $j$ is iterable. Moreover, for any $n \in \omega, j_{n}$ : $L\left(V_{\lambda+1}\right) \prec L\left(V_{\lambda+1}\right)$.

Theorem 4.2 states that $M_{n}=L\left(V_{\lambda+1}\right)$ for $n<\omega$, but $M_{\omega}$ is definitively different. The key point is that $j_{0, \omega}(\operatorname{crt}(j))=\lambda$, so many characteristics of the critical point of $j$ are transferred by elementarity to $\lambda$ in $M_{\omega}$. For example, in $L\left(V_{\lambda+1}\right), \operatorname{crt}(j)$ is measurable and there is a well-ordering of $V_{\lambda}$, therefore $\lambda$ is measurable in $M_{\omega}$ and there is a well-ordering of $V_{j_{0, \omega}(\lambda)}^{M_{\omega}}=V_{j_{0, \omega}(\lambda)} \cap M_{\omega}$ in $M_{\omega}$. In particular, also $V_{\lambda+1}^{M_{\omega}}=V_{\lambda+1} \cap M_{\omega}$ is well-ordered in $M_{\omega}$, and $V_{j_{0, \omega}(\lambda)} \cap M_{\omega} \vDash \mathrm{AC}$. This last point will be important in the proof of Theorem 4.4 below.

However, adding again the critical sequence to $M_{\omega}$ makes it a little more similar to the original $L\left(V_{\lambda+1}\right)$ : 
Theorem 4.3 (Generic Absoluteness). Let $j: L\left(V_{\lambda+1}\right) \prec L\left(V_{\lambda+1}\right)$ with $\operatorname{crt}(j)<\lambda$ be a proper elementary embedding. Let $\left\langle\kappa_{n}: n \in \omega\right\rangle$ be the critical sequence of $j$ and let $\left(M_{\omega}, j_{\omega}\right)$ be the $\omega$-th iterate of $j$. Then for all $\alpha<\lambda$ there exists an elementary embedding

$$
\pi: L_{\alpha}\left(M_{\omega}\left[\left\langle\kappa_{n}: n \in \omega\right\rangle\right] \cap V_{\lambda+1}\right) \prec L_{\alpha}\left(V_{\lambda+1}\right)
$$

such that $\pi \uparrow \lambda$ is the identity.

The previous theorem is Theorem 135 in [18] by Woodin.

Theorem 4.4. Suppose I0. Then there exists a generic extension $V[G]$ of $V$ such that $V[G] \vDash \exists \gamma \exists i: V_{\gamma+1} \prec V_{\gamma+1} \wedge 2^{\gamma}>\gamma^{+}$.

Proof. Let $j: L\left(V_{\lambda+1}\right) \prec L\left(V_{\lambda+1}\right)$ witness I0 in $V$. By Lemma 2.2 we can suppose that $j$ is proper, and by Corollary 3.9 we can suppose that for all regular cardinals $\kappa, 2^{\kappa}=\kappa^{++}$. Let $\left\langle\kappa_{n}: n \in \omega\right\rangle$ be the critical sequence of $j$. Let $\left(M_{\omega}, j_{\omega}\right)$ be the $\omega$-th iterate of $j$. Then, by elementarity, since $L\left(V_{\lambda+1}\right) \vDash$ "for all regular cardinals $\kappa<\lambda, 2^{\kappa}=\kappa^{++}$", $\lambda$ is regular in $M_{\omega}$ and $\lambda<j_{0, \omega}(\lambda), M_{\omega} \vDash$ "there exists a bijection between $\mathcal{P}(\lambda)$ and $\lambda^{++}$".

Let $U=\left\{X \subseteq \kappa_{0}: \kappa_{0} \in j(X)\right\}$ be the measure on $\kappa_{0}$ derived from $j$ and let $\mathbb{P}$ be the Prikry forcing on $\kappa_{0}$ with measure $U$. Then $j_{0, \omega}(\mathbb{P})$ is the Prikry forcing on $\lambda$ with measure $j_{0, \omega}(U)$.

Claim 4.5. $\left\langle\kappa_{n}: n \in \omega\right\rangle$ is generic for $j_{0, \omega}(\mathbb{P})$ over $M_{\omega}$.

Proof of Claim. We use the Mathias characterization of genericity for Prikry forcing [14], i.e., we prove that for any $A \in j_{0, \omega}(U)$, the set $\left\langle\kappa_{n}: n \in \omega\right\rangle \backslash A$ is finite. The characterization needs the Axiom of Choice, but works also in this setting, as $V_{j_{0, \omega}(\lambda)} \cap M_{\omega} \vDash \mathrm{AC}$. First, note that if $A \in j_{0, n}(U)$, then $\kappa_{n} \in j_{n, \omega}(A)$ : by definition of $U$ and elementarity $A \in j_{0, n}(U)$ iff $\kappa_{n} \in j_{n}(A)$; the critical point of $j_{n+1, \omega}$ is $\kappa_{n+1}$, so by elementarity

$$
\kappa_{n}=j_{n+1, \omega}\left(\kappa_{n}\right) \in j_{n+1, \omega}\left(j_{n}(A)\right)=j_{n, \omega}(A) .
$$

Now, let $A \in j_{0, \omega}(U)$. There exists $n \in \omega$ and $\bar{A} \in L\left(V_{\lambda+1}\right)$ such that $A=$ $j_{n, \omega}(\bar{A})$, and by elementarity $\bar{A} \in j_{0, n}(U)$ and, more generally, $j_{n, n+i}(\bar{A}) \in$ $j_{0, n+i}(U)$. So for any $i \in \omega, \kappa_{n+i} \in j_{n+i, \omega}\left(j_{n, n+i}(\bar{A})\right)=A$.

Because of the claim we can use the usual properties of Prikry forcing:

Claim 4.6. $M_{\omega}\left[\left\langle\kappa_{n}: n \in \omega\right\rangle\right] \vDash$ there exists a bijection from $2^{\lambda}$ to $\lambda^{++}$. 
Proof of Claim. Note that, even if $M_{\omega}\left[\left\langle\kappa_{n}: n \in \omega\right\rangle\right] \not \models A C$, we have in fact that $V_{j_{0, \omega}(\lambda)} \cap M_{\omega} \vDash A C$, therefore no special care is needed in this respect. Since Prikry forcing does not add bounded subsets of $\lambda, V_{\lambda} \cap M_{\omega}=$ $V_{\lambda} \cap M_{\omega}\left[\left\langle\kappa_{n}: n \in \omega\right\rangle\right]$, therefore for any name $\tau$ for a subset of $V_{\lambda} \cap M_{\omega}$, we can suppose that $\operatorname{dom}(\tau) \subseteq\left\{\check{a}: a \in V_{\lambda} \cap M_{\omega}\right\}$. Prikry forcing is $\lambda^{+}$-c.c., so there are only

$$
\left(2^{\lambda}\right)^{M_{\omega}}=\left(\lambda^{++}\right)^{M_{\omega}}=\left(\lambda^{++}\right)^{M_{\omega}\left[\left\langle\kappa_{n}: n \in \omega\right\rangle\right]}
$$

possible nice names for subsets of $V_{\lambda} \cap M_{\omega}$, and this proves the claim.

This proves that $\lambda$ in $M_{\omega}\left[\left\langle\kappa_{n}: n \in \omega\right\rangle\right]$ has the desired properties, and we will use generic absoluteness (Theorem 4.3) to prove the existence of the I1-elementary embedding.

Consider $j \uparrow V_{\lambda+1}: V_{\lambda+1} \prec V_{\lambda+1}$. We can define it as

$$
\left(j \uparrow V_{\lambda+1}\right)(a)=\bigcup_{n \in \omega}\left(j \uparrow V_{\lambda}\right)\left(a \cap V_{\kappa_{n}}\right),
$$

using only $j \uparrow V_{\lambda}$ and the critical sequence as parameters, both elements of $V_{\lambda+1}$, so $j \uparrow V_{\lambda+1} \in L_{1}\left(V_{\lambda+1}\right)$ and $L_{1}\left(V_{\lambda+1}\right) \vDash \exists i: V_{\lambda+1} \prec V_{\lambda+1}$. By generic absoluteness (Theorem 4.3) then

$$
L_{1}\left(M_{\omega}\left[\left\langle\kappa_{n}: n \in \omega\right\rangle\right] \cap V_{\lambda+1}\right) \vDash \exists i: V_{\lambda+1} \prec V_{\lambda+1},
$$

and this is enough, since $L_{1}\left(M_{\omega}\left[\left\langle\kappa_{n}: n \in \omega\right\rangle\right] \cap V_{\lambda+1}\right)$ computes correctly the satisfaction relation of

$$
M_{\omega}\left[\left\langle\kappa_{n}: n \in \omega\right\rangle\right] \cap V_{\lambda+1}=\left(V_{\lambda+1}\right)^{M_{\omega}\left[\left\langle\kappa_{n}: n \in \omega\right\rangle\right]}
$$

and

$$
L_{1}\left(M_{\omega}\left[\left\langle\kappa_{n}: n \in \omega\right\rangle\right] \cap V_{\lambda+1}\right) \subseteq M_{\omega}\left[\left\langle\kappa_{n}: n \in \omega\right\rangle\right]
$$

Therefore

$$
M_{\omega}\left[\left\langle\kappa_{n}: n \in \omega\right\rangle\right] \vDash \exists i: V_{\lambda+1} \prec V_{\lambda+1} .
$$

By reflecting $j_{0, \omega}$ we will have the desired generic extension: from what we proved above

$$
M_{\omega} \vDash \exists p \in j_{0, \omega}(\mathbb{P}) p \Vdash_{j_{0, \omega}(\mathbb{P})} \exists i:\left(V_{\check{\lambda}+1}\right) \prec\left(V_{\check{\lambda}+1}\right) \wedge 2^{\check{\lambda}}=\check{\lambda}^{++}
$$

therefore by elementarity of $j_{0, \omega}$

$$
L\left(V_{\lambda+1}\right) \vDash \exists p \in \mathbb{P} p \Vdash_{\mathbb{P}} \exists i:\left(V_{\check{\kappa}_{0}+1}\right) \prec\left(V_{\check{\kappa}_{0}+1}\right) \wedge 2^{\check{\kappa}_{0}}=\check{\kappa}_{0}^{++} .
$$

Let $G$ be any generic such that $p$ is as above and $p \in G$. Then in $L\left(V_{\lambda+1}\right)[G]$, and therefore in $V[G], \exists i: V_{\kappa_{0}+1} \prec V_{\kappa_{0}+1}$ and $2^{\kappa_{0}}=\kappa_{0}^{++}$. 
The proof does not depend specifically on $2^{\lambda}=\lambda^{++}$, therefore it yields a more general theorem.

Theorem 4.7 (Main). Suppose there exists $j: L\left(V_{\lambda+1}\right) \prec L\left(V_{\lambda+1}\right)$ with $\operatorname{crt}(j)<\lambda$. Then for every Easton function $E$ such that $E \uparrow \lambda$ is definable over $V_{\lambda}$, there is a generic extension $V[G]$ of $V$ that satisfies

$$
\exists \gamma<\lambda \exists i: V_{\gamma+1} \prec V_{\gamma+1}+2^{\gamma}=E(\gamma) .
$$

In particular, for any $\delta<\lambda$, there is a generic extension $V[G]$ of $V$ that satisfies

$$
\exists \gamma<\lambda \exists i: V_{\gamma+1} \prec V_{\gamma+1}+2^{\kappa}=\kappa^{+\delta+1}
$$

holds for all regular $\kappa<\gamma$ and also for $\kappa=\gamma$.

We make a short comment regarding the meaning of "larger", in the expression "one large cardinal is larger than the second one". Over the years the meaning of this sentence grew more and more ambiguous: it is mostly used to indicate the consistency strength of a large cardinal hypothesis, but it sometimes implies that the least cardinal which is large in the first sense is larger than the least cardinal which is large in the second sense with respect to the cardinal order. While at the beginning the two concepts coincided, during the exploration of the upper part of the large cardinal hierarchy the two concepts often differed completely. Theorem 4.4, coupled with Solovay's Theorem 3.10, gives us another such example.

Theorem 4.8. Suppose there exists $j: L\left(V_{\lambda+1}\right) \prec L\left(V_{\lambda+1}\right)$ with $\operatorname{crt}(j)<\lambda$. Then it is consistent that there exists $\gamma$ and $j: V_{\gamma+1} \prec V_{\gamma+1}$ and that every strongly compact cardinal is larger than $\gamma$.

\section{Consistency of hypotheses stronger than I1 and the negation of GCH}

In the proof of Theorem 4.4. Theorem 4.3 was greatly underutilized: the case $\alpha=1$, the one we considered, gave us a result related to I1, but in fact we can consider larger $\alpha$. This will give us consistency results on hypotheses stronger than I1, such as the existence of $j: L_{\alpha}\left(V_{\lambda+1}\right) \prec L_{\alpha}\left(V_{\lambda+1}\right)$, for $\alpha<\lambda$. These unnamed hypotheses have been proven by Laver [12], [13] to be strictly stronger than I1 and strictly weaker than I0.

It is also worthy not to stop at the bound given by Theorem 4.3 . There is no evidence that it is optimal. On the contrary, there are hints that generic absoluteness could possibly be extended, and Woodin outlined such situations in [18] (see for example Lemma 130 and Remark 139). 
Definition 5.1. Suppose $I 0(\lambda)$ and let $\alpha<\Theta^{L\left(V_{\lambda+1}\right)}$. We say that generic absoluteness holds at $\alpha$ if for any proper $j: L\left(V_{\lambda+1}\right) \prec L\left(V_{\lambda+1}\right)$ with $\operatorname{crt}(j)<$ $\lambda$ there exists

$$
\pi: L_{\alpha}\left(M_{\omega}\left[\left\langle\kappa_{n}: n \in \omega\right\rangle\right] \cap V_{\lambda+1}\right) \prec L_{\alpha}\left(V_{\lambda+1}\right)
$$

such that $\pi \uparrow \lambda$ is the identity, where $\left\langle\kappa_{n}: n \in \omega\right\rangle$ is the critical sequence of $j$ and $M_{\omega}$ is the model of its $\omega$-th iterate.

Theorem 4.3 states, therefore, that generic absolutess holds at any $\alpha<\lambda$.

The study of the structure of the sets $L_{\alpha}\left(V_{\lambda+1}\right)$ for $\alpha<\Theta^{L\left(V_{\lambda+1}\right)}$ made by Laver in [13] gives us the tools to approach Theorem 4.7 under stronger hypotheses.

Definition 5.2 ([13]). Let $\lambda$ be a cardinal and let $\alpha<\Theta^{L\left(V_{\lambda+1}\right)}$. Then $\alpha$ is good iff every element of $L_{\alpha}\left(V_{\lambda+1}\right)$ is definable in $L_{\alpha}\left(V_{\lambda+1}\right)$ from an element in $V_{\lambda+1}$.

The successor of a good ordinal is a good ordinal: let $\alpha$ be good; the largest ordinal in $L_{\alpha+1}\left(V_{\lambda+1}\right)$ is $\lambda+1+\alpha$, therefore $\alpha$ is definablein $L_{\alpha+1}\left(V_{\lambda+1}\right)$ with $\lambda$ as a parameter, so $L_{\alpha}\left(V_{\lambda+1}\right)$ is definable in $L_{\alpha+1}\left(V_{\lambda+1}\right)$ with $\lambda$ as a parameter. But every element in $L_{\alpha+1}\left(V_{\lambda+1}\right)$ is definable using $L_{\alpha}\left(V_{\lambda+1}\right)$ and elements of $L_{\alpha}\left(V_{\lambda+1}\right)$ (which in turn are definable with parameters from $V_{\lambda+1}$ ), and therefore $\alpha+1$ is good. This proves that the natural numbers are good. But also $\omega$ is good: every element of $L_{\omega}\left(V_{\lambda+1}\right)$ is in some $L_{n}\left(V_{\lambda+1}\right)$, $n \in V_{\lambda+1}$ and $n$ is good for every $n$. Following the same line of reasoning, every ordinal up to $\lambda$ is good, and considering that all ordinals less than $\lambda^{+}$ are coded as subsets of $\lambda$, and therefore in $V_{\lambda+1}$, every ordinal up to $\lambda^{+}$is good.

On the other side, non-good ordinals exist. The definition of good ordinal is restricted to ordinals strictly less than $\Theta^{L\left(V_{\lambda+1}\right)}$ because larger ones are trivially not good: if $x$ is definable with a parameter, then it is uniquely determined by its definition, therefore for any $\alpha$ there exists in $L\left(V_{\lambda+1}\right), \pi: V_{\lambda+1} \rightarrow$ $G_{\alpha}=\left\{x \in L_{\alpha}\left(V_{\lambda+1}\right): L_{\alpha}\left(V_{\lambda+1}\right) \vDash x\right.$ is definable from an element in $\left.V_{\lambda+1}\right\}$; then if $\Theta^{L\left(V_{\lambda+1}\right)} \subseteq L_{\alpha}\left(V_{\lambda+1}\right), G_{\alpha}$ must be strictly contained in $L_{\alpha}\left(V_{\lambda+1}\right)$, by definition of $\Theta^{L\left(V_{\lambda+1}\right)}$. But non-good ordinals exist also below $\Theta^{L\left(V_{\lambda+1}\right)}$ : define $L_{\gamma}\left(V_{\lambda+1}\right)$ as the collapse of the Skolem closure of $V_{\lambda+1}$ in $L_{\Theta}{ }^{L\left(V_{\lambda+1}\right)}\left(V_{\lambda+1}\right)$; as $L_{\Theta^{L\left(V_{\lambda+1}\right)}}\left(V_{\lambda+1}\right) \vDash \exists x x$ not definable from an element in $V_{\lambda+1}$, by elementarity the same must be true in $L_{\gamma}\left(V_{\lambda+1}\right)$, as the collapse does not collapse $V_{\lambda+1}$, therefore $\gamma$ is not good.

One can ask how many good ordinals there are.

Lemma $5.3([13])$. Let $\lambda$ be a strong limit cardinal. Then the good ordinals are unbounded in $\Theta^{L\left(V_{\lambda+1}\right)}$. 
Therefore assuming $\alpha$ good is in most cases a reasonable choice.

By the usual condensation argument, if $\alpha<\Theta^{L\left(V_{\lambda+1}\right)}$ and $i: L_{\alpha}\left(V_{\lambda+1}\right) \rightarrow$ $L_{\alpha}\left(V_{\lambda+1}\right)$ then $i \in L_{\Theta}^{L\left(V_{\lambda+1}\right)}\left(V_{\lambda+1}\right)$. If $\alpha$ is good, however, it is possible to be much more precise:

Lemma $5.4([13])$. Let $\lambda$ and $\alpha$ be such that $\alpha$ is good and there exists $i: L_{\alpha}\left(V_{\lambda+1}\right) \prec L_{\alpha}\left(V_{\lambda+1}\right)$ with $\operatorname{crt}(i)<\lambda$. Then $i$ is induced by $i \uparrow V_{\lambda}$, and therefore $i \in L_{\alpha+1}\left(V_{\lambda+1}\right)$.

Lemma 5.5 (Woodin). Let $\lambda$ be a cardinal. If there exists $j: L_{\Theta^{L\left(V_{\lambda+1}\right)}}\left(V_{\lambda+1}\right) \prec$ $L_{\Theta}^{L\left(V_{\lambda+1}\right)}\left(V_{\lambda+1}\right)$, then for any $\alpha<\Theta^{L\left(V_{\lambda+1}\right)}$ there exists an $i: L_{\alpha}\left(V_{\lambda+1}\right) \prec$ $L_{\alpha}\left(V_{\lambda+1}\right)$.

Proof. Suppose it is false. Then there is a counterexample $\alpha$ such that every $i: L_{\alpha}\left(V_{\lambda+1}\right) \rightarrow L_{\alpha}\left(V_{\lambda+1}\right)$ is not an elementary embedding. All such $i$ 's are in $L_{\Theta}^{L\left(V_{\lambda+1}\right)}\left(V_{\lambda+1}\right)$ (see remark before Lemma 5.4), therefore $L_{\Theta^{L\left(V_{\lambda+1}\right)}}\left(V_{\lambda+1}\right) \vDash$ $\exists \alpha$ ( $\alpha$ is a counterexample). Let $\alpha_{0}$ be the least counterexample. Then $\alpha_{0}$ is definable in $L_{\Theta}^{L\left(V_{\lambda+1}\right)}\left(V_{\lambda+1}\right)$ and $j\left(\alpha_{0}\right)=\alpha_{0}$. Then $j \uparrow L_{\alpha_{0}}\left(V_{\lambda+1}\right)$ is as in the lemma, contradiction.

The previous lemmas suffice to prove a more general version of Theorem 4.7.

Theorem 5.6. Suppose that there exists $j: L\left(V_{\lambda+1}\right) \prec L\left(V_{\lambda+1}\right)$ with $\operatorname{crt}(j)=$ $\kappa_{0}<\lambda$ and that generic absoluteness holds at $\alpha+1$, with $\alpha$ good and such that $\alpha=j_{0, \omega}(\beta)$ for some $\beta$. Then there exists a generic extension that satisfies

$$
\exists i: L_{\beta}\left(V_{\kappa_{0}+1}\right) \prec L_{\beta}\left(V_{\kappa_{0}+1}\right)+2^{\kappa_{0}}=\left(2^{\kappa_{0}}\right)^{V} .
$$

Proof. The key point is that by Lemma 5.5 there exists $i: L_{\alpha}\left(V_{\lambda+1}\right) \prec$ $L_{\alpha}\left(V_{\lambda+1}\right)$ and by Lemma 5.4, $i \in L_{\alpha+1}\left(V_{\lambda+1}\right)$, therefore

$$
L_{\alpha+1}\left(V_{\lambda+1}\right) \vDash \exists i: L_{\alpha}\left(V_{\lambda+1}\right) \prec L_{\alpha}\left(V_{\lambda+1}\right) \text {. }
$$

Let $\left(2^{\kappa_{0}}\right)^{V}=\eta$. Using the same notation as in the proof of Theorem 4.4 . by the elementarity of $j_{0, \omega}, M_{\omega} \vDash$ there exists a bijection between $\mathcal{P}(\lambda)$ and $j_{0, \omega}(\eta)$, and this is true also in $M_{\omega}\left[\left\langle\kappa_{n}: n \in \omega\right\rangle\right]$. By generic absoluteness at $\alpha+1$ there exists an elementary embedding

$$
\pi: L_{\alpha+1}\left(M_{\omega}\left[\left\langle\kappa_{n}: n \in \omega\right\rangle\right] \cap V_{\lambda+1}\right) \prec L_{\alpha+1}\left(V_{\lambda+1}\right) .
$$

Note that $\pi(\alpha)=\alpha$, as $\alpha$ is definable in both models. Since

$$
L_{\alpha+1}\left(V_{\lambda+1}\right) \vDash \exists i: L_{\alpha}\left(V_{\lambda+1}\right) \prec L_{\alpha}\left(V_{\lambda+1}\right),
$$




$$
L_{\alpha+1}\left(M_{\omega}\left[\left\langle\kappa_{n}: n \in \omega\right\rangle\right] \cap V_{\lambda+1}\right) \vDash \exists i: L_{\alpha}\left(V_{\lambda+1}\right) \prec L_{\alpha}\left(V_{\lambda+1}\right),
$$

and therefore

$$
M_{\omega}\left[\left\langle\kappa_{n}: n \in \omega\right\rangle\right] \vDash \exists i: L_{\alpha}\left(V_{\lambda+1}\right) \prec L_{\alpha}\left(V_{\lambda+1}\right) \wedge 2^{\lambda}=j_{0, \omega}(\eta) .
$$

This means that

$$
M_{\omega} \vDash \exists p \in j_{0, \omega}(\mathbb{P}) p \Vdash \exists i: L_{\check{\alpha}}\left(V_{\check{\lambda}+1}\right) \prec L_{\check{\alpha}}\left(V_{\check{\lambda}+1}\right) \wedge 2^{\check{\lambda}}=j_{0, \omega}(\check{\eta})
$$

and by the elementarity of $j_{0, \omega}$

$$
L\left(V_{\lambda+1}\right) \vDash \exists p \in \mathbb{P} p \Vdash \exists i: L_{\check{\beta}}\left(V_{\check{\kappa}_{0}+1}\right) \prec L_{\check{\beta}}\left(V_{\check{\kappa}_{0}+1}\right) \wedge 2^{\check{\kappa}_{0}}=\check{\eta}
$$

and this proves the theorem.

Corollary 5.7. Suppose $I O(\lambda)$. Let $E$ be an Easton function such that $E \uparrow \lambda$ is definable over $V_{\lambda}$ and let $\alpha<\lambda$. Then there exists $j: L\left(V_{\lambda+1}\right) \prec L\left(V_{\lambda+1}\right)$ with $\alpha<\operatorname{crt}(j)<\lambda$ and a generic extension in which

$$
\exists i: L_{\alpha}\left(V_{\operatorname{crt}(j)}\right) \prec L_{\alpha}\left(V_{\operatorname{crt}(j)}\right)+2^{\operatorname{crt}(j)}=E(\operatorname{crt}(j))
$$

holds.

Proof. By Theorem 3.2, we can start with a generic extension in which $2^{\kappa}=$ $E(\kappa)$ for any $\kappa$ regular. Fix a $j$ that witnesses $\mathrm{I} 0(\lambda)$ and such that $\operatorname{crt}(j)$ is bigger than $\alpha$. Then $j(\alpha)=\alpha$ and $j_{0, \omega}(\alpha)=\alpha$. Since generic absoluteness holds below $\lambda$ and $\alpha$ is good, by Theorem 5.6 the corollary holds.

Theorem 4.3 states that generic absoluteness holds for $\alpha$ less than $\lambda$, therefore this is the bound for Theorem 5.6 for now. Even if a generalization of generic absoluteness could be proved, there would still be questions to answer. Let $\mathrm{I} 0(\lambda)$ and $\mathrm{I} 1(\lambda)$ be the the corresponding hypotheses with fixed $\lambda$. While we used I0 for the consistency strength of $\exists \lambda \operatorname{I} 1(\lambda)+$ the failure of $\mathrm{GCH}$ at $\lambda$, it is not known whether this is optimal.

Question 1. Does Con $(\mathrm{ZFC}+I 1)$ imply $\operatorname{Con}\left(\mathrm{ZFC}+\exists \lambda\left(I 1(\lambda) \wedge 2^{\lambda}>\lambda^{+}\right)\right)$?

Theorem 4.7 is limited also in a further direction: in the theorem the behaviour at $\lambda$ of the power function is controlled by the Easton function on the regular cardinals below it. But this seems not a necessary condition, as in Theorem $3.11 \lambda$ is in fact the first cardinal at which GCH fails. Therefore we can ask:

Question 2. Is ZFC $+\exists \lambda\left(I 1(\lambda) \wedge 2^{\lambda}>\lambda^{+} \wedge \forall \kappa<\lambda 2^{\kappa}=\kappa^{+}\right)$consistent? If so, what is its consistency strength? 
Another way to improve the results in this paper would be to approach the consistency of $\mathrm{I} 0(\lambda)+$ the failure of $\mathrm{GCH}$ at $\lambda$.

Question 3. Is ZFC $+\exists \lambda\left(I 0(\lambda) \wedge 2^{\lambda}>\lambda^{+}\right)$consistent? If so, what is its consistency strength?

There is a result of Woodin that indicates a direction for a solution for Question 3 and can be seen as a corollary to Theorem 5.6 .

Corollary 5.8 (Woodin). Suppose that generic absoluteness holds at any $\alpha<\Theta^{L\left(V_{\lambda+1}\right)}$. Then

$$
\operatorname{Con}\left(Z F C+\exists \lambda\left(I 0(\lambda)+2^{\lambda} \text { large }\right)\right) .
$$

Sketch of proof. As generic absoluteness holds for any $\alpha<\Theta^{L\left(V_{\lambda+1}\right)}$, for any such $\alpha$,

$$
\pi^{-1}\left(j\left\lceil L_{\alpha}\left(V_{\lambda+1}\right)\right):\left(L_{\alpha}\left(V_{\lambda+1}\right)\right)^{M_{\omega}\left[\left\langle\kappa_{i}: i \in \omega\right\rangle\right]} \prec\left(L_{\alpha}\left(V_{\lambda+1}\right)\right)^{M_{\omega}\left[\left\langle\kappa_{i}: i \in \omega\right\rangle\right]},\right.
$$

and therefore $j^{*}=\bigcup_{\alpha<\Theta^{L\left(V_{\lambda+1}\right)}} \pi^{-1}\left(j\left\lceil L_{\alpha}\left(V_{\lambda+1}\right)\right)\right.$ is an elementary embedding from $\left(L_{\Theta^{L\left(V_{\lambda+1}\right)}}\left(V_{\lambda+1}\right)\right)^{M_{\omega}\left[\left\langle\kappa_{i}: i \in \omega\right\rangle\right]}$ to itself. Define $U_{j^{*}}$ as in Lemma 2.2. Then the ultrapower of $\left(L\left(V_{\lambda+1}\right)\right)^{M_{\omega}\left[\left\langle\kappa_{i}: i \in \omega\right\rangle\right]}$ is well-founded, and this proves I0 in $M_{\omega}\left[\left\langle\kappa_{i}: i \in \omega\right\rangle\right]$. As with Theorem 5.6, it is possible to make $2^{\lambda}$ large in $M_{\omega}\left[\left\langle\kappa_{i}: i \in \omega\right\rangle\right]$, and because of the elementarity of $j_{0, \omega}$ the corollary is proved.

The consistency strength of such hypothesis is not yet known. A different road would be to extend generic absoluteness in another direction. In [18] Woodin introduced new hypotheses even stronger than I0, and it would be natural to prove an analog of Theorem 4.3 in that setting as a strategy for answering Question 3 affirmatively. However this seems difficult and therefore Question 3 remains a compelling challenge.

\section{References}

[1] P. Corazza, The wholeness axiom and Laver sequences. Annals of Pure and Applied Logic 105 (2000), 157-260.

[2] P. Corazza, Lifting elementary embeddings $j: V_{\lambda} \rightarrow V_{\lambda}$. Archive for Mathematical Logic 46 (2007), 61-72.

[3] J. Cummings, M. Foreman, Diagonal Prikry extensions. Journal of Symbolic Logic 75 (2010), 1383-1402. 
[4] W. B. Easton, Powers of regular cardinals. Annals of Mathematical Logic 1 (1970), 139-178.

[5] S.-D. Friedman, Large cardinals and L-like universes. Set Theory: Recent Trends and Applications (A. Andretta editor). Quaderni di Matematica 17 (2006), 93-110.

[6] M. Gitik, Blowing up the power of a singular cardinal - wider gaps. Annals of Pure and Applied Logic 116 (2002), 1-38.

[7] M. Gitik, Prikry-type forcings. Handbook of Set Theory (M. Foreman and A. Kanamori editors), Springer Netherlands 2010, 1351-1447.

[8] J. Hamkins, Fragile measurability. Journal of Symbolic Logic 59 (1994), $262-282$.

[9] F. Hausdorff, Grundzüge einer Theorie der geordneten Mengen. Mathematische Annalen 65 (1908), 435-505.

[10] A. Kanamori, The Higher Infinite. Springer, Berlin 1994.

[11] K. Kunen, Elementary embeddings and infinite combinatorics. Journal of Symbolic Logic 36 (1971), 407-413.

[12] R. Laver, Implications between strong large cardinal axioms. Annals of Pure and Applied Logic 90 (1997), 79-90.

[13] R. Laver, Reflection of elementary embedding axioms on the $L\left[V_{\lambda+1}\right]$ hierarchy. Annals of Pure and Applied Logic 107 (2001), 227-238.

[14] A. R. D. Mathias, On sequences generic in the sense of Prikry. Journal of the Australian Mathematical Society 15 (1973), 409-414.

[15] D. Scott, Measurable cardinals and constructible sets. Bulletin de l'Académie Polonaise des Sciences, Série des Sciences Mathématiques, Astronomiques et Physiques 9 (1961), 521-524.

[16] J. Silver, On the singular cardinals problem. Proceedings of the International Congress of Mathematicians, Vancouver 1974, 265-268

[17] R. Solovay, Strongly compact cardinals and the GCH. Proceedings of the Tarski Symposium, Proceedings of Symposia in Pure Mathematics 25, Providence, American Mathematical Society, 1974, 365-372.

[18] W. H. Woodin, Suitable extender models II: Beyond $\omega$-huge. Journal of Mathematical Logic 11 (2011), 115-436. 